\title{
Risk Sharing Across Communities
}

\author{
by Yann Bramoullé and Rachel Kranton ${ }^{1}$
}

January 2007

This paper studies cross-community risk sharing. There is now a large body of theoretical and empirical work on informal insurance, where people mitigate risk by sharing income. A consistent empirical finding is that risk-sharing is not complete within villages, often the observed sets of individuals. ${ }^{2}$ One reason, researchers suspect, is that risk-sharing does not take place at the village level, but between individuals and families. ${ }^{3}$ We build a theoretical model where risksharing takes place between pairs of agents. There are idiosyncratic shocks to individual income and community-level shocks. We consider how the opportunity for cross-community links affects the shape and efficiency of risk sharing arrangements.

We find that when links across villages form, there can be less risk sharing within a village. Welfare is higher for those directly or indirectly connected across villages, but lower for those with no path connecting them to the other village. Overall, welfare can be higher. Thus, empirical findings that insurance within a village is not complete is not necessarily evidence of an inefficient pattern of risk-sharing relations. Rather, the finding is consistent with risk-sharing patterns that involve cross-community relations, and such patterns may yield higher aggregate welfare despite incomplete insurance within a village.

This paper makes two contributions. First, it demonstrates the importance of a network analysis. By examining pairwise incentives to form relations and the resulting pattern, we can see new risk sharing outcomes. Second, with this network model, we show how informal risk sharing across communities has both aggregate and distributional effects.

To study network outcomes, we posit a simple two stage model. First, we provide a benchmark model for risk-sharing for a given network. Then, working backwards, we consider incentives to form risk-sharing links. ${ }^{4}$ To illustrate: Consider a rural population and ask how people might link

\footnotetext{
${ }^{1}$ Bramoullé: Department of Economics, CIRPÉE and GREEN, Université Laval, Québec, QC, G1K 7P4, Canada; Kranton: Department of Economics, University of Maryland, College Park, MD, 20742, USA. Rachel Kranton thanks the National Science Foundation for financial support.

${ }^{2}$ For seminal contributions see Townsend (1994) and Udry (1995). Recently, see Dercon \& DeWeerdt (2006).

${ }^{3}$ See Fafchamps \& Lund (2003), Murgai et al. (2002), Townsend (1994, p. 541).

${ }^{4}$ Most previous theoretical literature studies the enforcement of risk-sharing agreements (e.g., Coate \& Ravallion (1993), Ligon \& Worrall (2002)). Bloch, Genicot, \& Ray (2006) studies enforcement when income is shared by
} 
with others - by marriage say - in order to establish a risk-sharing relation. (There is evidence in India, for example, that families arrange daughters' marriages to men in different villages to increase gains from risk sharing (Rosenzweig and Stark (1989)). Establishing this relation is costly, e.g., involving a dowry and marriage ceremony, and the relation commits the parties to future income sharing, say, due to a social norm or social punishment in case of non-sharing. The marriage pattern would then be our network.

We allow agents to form links both inside and outside of their communities. In our model, a link commits pairs to share equally future monetary holdings, and the end outcome corresponds to the highest level of insurance possible in a given network. Hence any divergence from efficiency comes from the decisions to form links. We ask when stable networks span communities and examine the welfare properties of such networks.

\section{A Model of Risk Sharing}

There are two villages, and the sets of individuals living in villages 1 and 2 are $V_{1}$ and $V_{2}$, respectively, where $\left|V_{1}\right|=m_{1}$ and $\left|V_{2}\right|=m_{2}$. Individual income is a random variable, and individuals are risk averse. For agent $i$ living in village $v$, income is the sum of three terms: $\widetilde{y}_{i}=\bar{y}+\widetilde{\varepsilon}_{i}+\widetilde{\mu}_{v}$, where $\widetilde{\varepsilon}_{i}$ is an idiosyncratic shock and $\widetilde{\mu}_{v}$ is a shock common to all agents of village $v{ }^{5}$ We assume the village shocks are i.i.d. with mean zero and variance $\sigma_{\mu}^{2}$, and the idiosyncratic shocks are i.i.d. with mean zero and variance $\sigma_{\varepsilon}^{2}$. The village and idiosyncratic shocks are also independently distributed. Agents have the same preferences, with an increasing and strictly concave utility function $v(y)$. While formal insurance is not available, to smooth income pairs of agents can transfer money after shocks are realized. To do so, two agents must establish a "link" which represents a relationship-specific investment allowing the pair to observe each others' monetary holdings and make tranfers. A link between a pair of agents in the same village costs each agent $c$. A link between two agents in different villages costs each agent $C$, where $C>c>0$. Our key assumptions are, then, (1) money transfers are possible only between pairs, (2) the links that allow these transfers must be made ex ante, (3) these links are costly,

${ }^{5}$ We use the word "village" throughout the paper, but it need not be taken literally. It is the common shock that differentiates agents in the population.
} 
and these costs cannot be shared. ${ }^{6}$

We represent links and network as follows: $g$ is an $n \times n$ matrix, where $g_{i j}=1$ when $i$ and $j$ have a link, and $g_{i j}=0$ otherwise. Income-sharing is mutual: $g_{i j}=g_{j i}$, and by convention, $g_{i i}=0$. There is a path between $i$ and $j$ if there exists a sequence of individuals $i_{1}, \ldots, i_{k}$ such that $g_{i i_{1}}=g_{i_{1} i_{2}}=\ldots=g_{i_{k} j}=1$. A subset of agents is connected if there is a path between any two agents in the subset. A component of the graph $g$ is a maximal connected subset. Components, then, provide a partition of the population. A graph is minimally connected when the removal of any link increases the number of components.

We first specify how linked individuals would share income. We then consider the formation of links.

\section{A. Risk Sharing in a Given Network}

Given a network $g$, how do pairs share realized income? We use the benchmark model developed in Bramoullé \& Kranton (2005). Suppose that after income is realized, linked pairs meet (sequentially and randomly) and share equally their current money holdings. ${ }^{7}$ With many rounds of such meetings, in the limit an individual's money holdings converge to the mean of realized income in his component, yielding the highest possible aggregate expected utility for this set of agents (Bramoullé \& Kranton (2005), Proposition 1).

With this benchmark, risk-sharing benefits depend only on the number of individuals from each village in a component. Let $n_{v}$ denote the number of people from village $v$ in a component. Ex-post income of an individual in a component containing $n_{1}$ and $n_{2}$ agents is then $\bar{y}+\frac{\sum_{i=1}^{n_{1}+n_{2}} \varepsilon_{i}}{n_{1}+n_{2}}+\frac{n_{1} \mu_{1}+n_{2} \mu_{2}}{n_{1}+n_{2}}$, where $\varepsilon_{i}$ is the realization of $i$ 's idiosyncratic shock and $\mu_{v}$ the realization of village $v$ 's shock. Let the expected utility of individual $i$ in a component containing $n_{1}$ and $n_{2}$ be $u\left(n_{1}, n_{2}\right)$ :

$$
u\left(n_{1}, n_{2}\right)=E v\left(\bar{y}+\frac{\sum_{i=1}^{n_{1}+n_{2}} \varepsilon_{i}}{n_{1}+n_{2}}+\frac{n_{1} \mu_{1}+n_{2} \mu_{2}}{n_{1}+n_{2}}\right)
$$

\footnotetext{
${ }^{6}$ Some costs, e.g., the time incurred to build a relation, are not easy to compensate. But link costs could be seen as another element of income to be shared by a pair. See discussion in Bramoullé \& Kranton (2005).

${ }^{7}$ For example, consider an agricultural setting and at the end of the growing season, farmers' incomes are realized. They then visit with their linked partners and share incomes. Then visit linked relations again, and so on, until the beginning of the following season.
} 
where the expectation is taken over all realizations of the village shocks and all the indiosyncratic shocks of the individuals in the component

In the rest of the paper, for simplicity we consider a quadratic utility function $v(y) \equiv y-\lambda y^{2}$, where $\lambda$ is a positive parameter such that $\lambda<\frac{1}{2 y}$ for all values of $y$. This specification has the advantage that expected utility depends only on the mean and variance of individual's income: $E v(y)=v(E(y))-\lambda v a r(y),{ }^{8}$ and a higher $\lambda$ corresponds to greater risk-aversion. An individual in a component containing $n_{1}$ and $n_{2}$ agents then has expected utility

$$
u\left(n_{1}, n_{2}\right)=u_{0}+\left(1-\frac{1}{n_{1}+n_{2}}\right) \lambda \sigma_{\varepsilon}^{2}+\frac{2 n_{1} n_{2}}{\left(n_{1}+n_{2}\right)^{2}} \lambda \sigma_{\mu}^{2}
$$

where $u_{0} \equiv v(\bar{y})-\lambda\left(\sigma_{\varepsilon}^{2}+\sigma_{\mu}^{2}\right)$

The expected utility function gives us important insights into the costs and benefits of risk sharing outside one's own community. The first term, $u_{0}$, is the utility an individual obtains when he does not participate in any income sharing. The second term shows the benefits from sharing the idiosyncratic shock. It is always increasing in both $n_{1}$ and $n_{2}$, showing that it always beneficial to share this risk with more people, regardless of their village. The third term shows the benefits of sharing the village-level shock. This term is positive as long as $n_{1}>0$ and $n_{2}>0$. That is, it is better to have links across villages than to have no such links.

But the number and proportion of agents in each village matter. The largest gain from sharing the village-level shock occurs when equal numbers of agents from both villages are in the component; that is, for $n_{1}=n_{2}>0$. Any move away from equality hurts agents, as agents are subject to greater income variance. To see this, suppose $\tilde{\mu}_{v}$ is equal to $\mu$ or to $-\mu$ with probability $\frac{1}{2}$ each, and the idiosyncratic shock always take on zero value. Suppose $n_{1}=n_{2}=1$. With probability $\frac{1}{2}$, one village has the high shock and the other village has a low shock, and after income sharing, each individual has income $\bar{y}$. Now suppose $n_{2}=2>n_{1}=1$. When village 2 has the low shock, individuals have income $\bar{y}-\frac{\mu}{3}$, and when village 2 has the high shock, individuals have income $\bar{y}+\frac{\mu}{3}$. Income variance is higher.

Thus, agents can have a disincentive to link to more agents in their own village, since it can increase the risk from sharing the village shock. As we can see in the above example, this effect

\footnotetext{
${ }^{8}$ Similar properties would obtain from an exponential utility function and normally distributed incomes. The expected utility would be an increasing and concave transform of the one we consider in the paper.
} 
is not special to our utility function. Indeed, general utility functions could have an additional effect. Increases in expected utility from sharing the village level shock could reduce the marginal utility from sharing the idiosyncratic shock with yet another agent within a village. ${ }^{9}$

\section{B. Pairwise Stable Networks}

We ask when agents might form cross-village links, and whether these links can increase or decrease aggregate welfare. ${ }^{10}$ In our setting, in a network $g$, each agent $i$ earns

$$
U_{i}(g)=u\left(n_{1}, n_{2} ; s_{i}(g)\right)-\left[c \sum_{j s . t . i j \in V} g_{i j}\right]-\left[C \sum_{j \text { s.t.ij } \notin V} g_{i j}\right]
$$

where $s_{i}(g)$ denotes the component containing $i$ which determines $n_{1}$ and $n_{2}$ for $i$, and the notation $i j \in V$ indicates $i$ and $j$ are in the same village. We then have individual expected utility from risk sharing, minus the sum of an individual's link costs.

We consider networks that are pairwise stable (Jackson \& Wolinsky (1996)): In a pairwise stable network, no single agent can improve her payoffs by breaking a link, and, for any pair of unlinked agents, if one agent's payffs would increase from a link, the second agent would not (hence the link is not formed). Let $g+i j$ denote the graph $g$ with the addition of a link between agents $i$ and $j$, and let $g-i j$ denote a graph $g$ subtracting any link between agents $i$ and $j$. A risk-sharing network $g$ is pairwise stable if and only if

$$
\begin{aligned}
& \text { (1) } \forall i j \text { s.t } g_{i j}=0 \text { if } U_{i}(g+i j)>U_{i}(g) \text { then } U_{j}(g+i j)<U_{j}(g) \text {, } \\
& \text { and (2) } \forall i j \text { s.t } g_{i j}=1, U_{i}(g) \geq U_{i}(g-i j) \text {. }
\end{aligned}
$$

Observe, first, that all pairwise stable graphs have minimally connected components. If a component is not minimally connected, an agent can sever a link without affecting $n_{1}$ and $n_{2}$; benefits are unchanged and costs are lower. Second, if $C$ is sufficiently large, pairwise stable networks have only within-village links.

\footnotetext{
${ }^{9}$ In our specification, expected utility is additively separable in gains from sharing the idiosyncratic shock and the village shock.

${ }^{10}$ Bramoulle \& Kranton (2005) provides a full analysis of within-village link formation.
} 


\section{Within-Village Risk Sharing Networks}

Consider a setting where the two villages are the same size: $m_{1}=m_{2}=m$. (We discuss below $m_{1} \neq m_{2}$.) Consider a network that yields complete insurance within a village but provides no insurance against village level shocks. This network has two components - all agents in one village are in one component, and all agents in the other village are in the other component. We call this network a "full-insurance-segregated network." See the Figure below. To be stable, within-village link costs must be small so it is always beneficial for an individual to maintain a link to the last agent in the village: ${ }^{11}$

$$
\left(\frac{1}{m-1}-\frac{1}{m}\right) \lambda \sigma_{\varepsilon}^{2} \equiv \bar{c} \geq c
$$

This network is pairwise stable if and only if $c \leq \bar{c}$, and no two agents in different villages want to form a link to insure against the village-level shock, $\frac{\lambda}{2} \sigma_{\mu}^{2}+\frac{\lambda}{2 m} \sigma_{\varepsilon}^{2}<C$.

\section{Cross-Village Risk Sharing Networks}

We compare this network to one where some agents insure against the village level shocks. We ask: When complete insurance within a village is possible, is it also possible that people in a village be divided into two sets - one set is connected to agents in the other village, and one set is not? We ask when such a network is stable and who would benefits and who would lose from such an arrangement.

Consider in each village a subgroup of size $k$. Let these $k$ people be in the same component with $k$ people in the other village, where there is one cross-village link. We call these agents the "core," and suppose $k \leq n / 2{ }^{12}$ Let the remaining $m-k$ agents be in a single component in each village. We say these agents are in the "periphery." See the Figure below. This network is pairwise stable if and only if no pair within a village periphery wants to cut a link, and no pair within a village core wants to cut a link. These conditions are satisfied for $c \leq \bar{c}$. Second, (i) no two agents in the periphery have an incentive to form a cross-village link,

$$
\left(\frac{1}{m-k}\right) \frac{\lambda}{2} \sigma_{\varepsilon}^{2}+\frac{\lambda}{2} \sigma_{\mu}^{2}<C
$$

\footnotetext{
${ }^{11} \mathrm{~A}$ minimally connected component always contains an agent that has only one link. The neighbor of this agent has the lowest incentive to keep this link. Hence, this agent must have the incentive to maintain the link in order for the graph to be stable.

${ }^{12}$ Our results also hold when the cores are different sizes but the difference is not too large.
} 

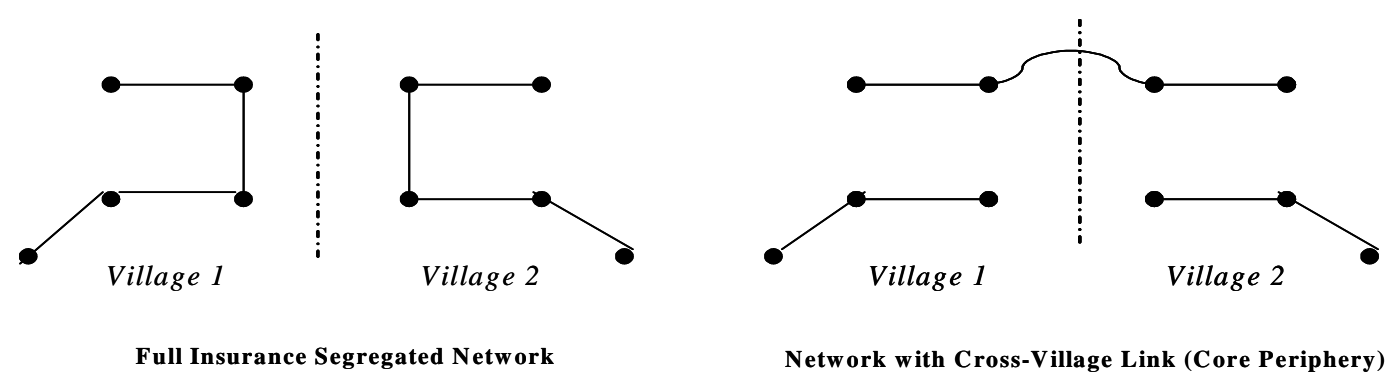

Figure 1: Networks with and without Cross-Village Link

(ii) the pair in the core with the cross-village link does not want to cut their link,

$$
\left(\frac{1}{k}\right) \frac{\lambda}{2} \sigma_{\varepsilon}^{2}+\frac{\lambda}{2} \sigma_{\mu}^{2} \geq C
$$

(iii) no agent in the core wants to form a link with an agent within the periphery of his own village, ${ }^{13}$

$$
\left(\frac{1}{k}-\frac{2}{m+k}\right) \frac{\lambda}{2} \sigma_{\varepsilon}^{2}-\left(\frac{m-k}{m+k}\right)^{2} \frac{\lambda}{2} \sigma_{\mu}^{2}<c,
$$

There are two forces at play in this last, key, condition. On one hand, an agent in the core would like to connect to an agent in his village in order to improve risk-sharing on his idiosyncratic shock - this is the positive term $\left(\frac{1}{k}-\frac{2}{m+k}\right) \frac{\lambda}{2} \sigma_{\varepsilon}^{2}$. On the other hand, this link would worsen risk-sharing on the village level shock. - this is the negative term $-\left(\frac{m-k}{m+k}\right)^{2} \frac{\lambda}{2} \sigma_{\mu}^{2}$. Clearly, if $\frac{\lambda}{2} \sigma_{\mu}^{2}$ is large enough, the latter effect dominates. When village level shocks have a high variance, individuals are less interested in creating within-village links. While they guard against the idiosyncratic shock, they increase the exposure to sharing large realizations of the village-level shock.

There exist ranges of costs $C$ and $c$ where both core-periphery networks and full-insurancesegregated networks are stable. First consider the condition on $C$. If no two agents in a periphery (assumed to be of size $k<m$ ) want to form a cross-village link, then no two agents in a fully connected village would want to do so either. ${ }^{14}$ Next consider the condition on $c$. When there

\footnotetext{
${ }^{13}$ Linking to an agent in the periphery in the other village is more costly. An agent in the periphery would gain more from connecting to the core than an agent in the core gains from connecting to an agent in the periphery when holds if $\lambda \sigma_{\mu}^{2}$ is not too low; precisely, if $\frac{\lambda}{2} \sigma_{\mu}^{2} \geq\left(\frac{1}{2 k}-\frac{1}{m-k}\right) \lambda \sigma_{\varepsilon}^{2}$.

${ }^{14}$ If $C>\frac{\lambda}{2} \sigma_{\mu}^{2}+\left(\frac{1}{2(m-k)}\right) \lambda \sigma_{\varepsilon}^{2}$, then $C>\frac{\lambda}{2} \sigma_{\mu}^{2}+\left(\frac{1}{2 m}\right) \lambda \sigma_{\varepsilon}^{2}$.
} 
is a large enough variance on the village level shock, there is a range of $c$ where both types of networks are stable. That is, combining $\bar{c} \geq c$ and (3) we have:

$$
\left(\frac{1}{m(m-1)}\right) \lambda \sigma_{\varepsilon}^{2} \geq c>\left(\frac{m-k}{2(m+k)}\right) \lambda \sigma_{\varepsilon}^{2}-\left(\frac{m-k}{m+k}\right)^{2} \frac{\lambda}{2} \sigma_{\mu}^{2}
$$

which is clearly satisfied for $\sigma_{\mu}^{2}$ sufficiently large. ${ }^{15}$

\section{E. Welfare Comparison: Does Cross-Village Risk Sharing Improve Outcomes?}

Is welfare higher when (some) agents share village level shocks? When both a core-periphery network is stable and a full-insurance-segregated network is stable, which yields higher welfare, and who benefits and who loses from a cross-village link.?

Let the welfare of a graph be the sum of all individuals' payoffs. The welfare of a core-periphery network, $W_{C P}$, is the sum of expected utility of core agents, $2 k\left[u_{0}+\left(1-\frac{1}{2 k}\right) \lambda \sigma_{\varepsilon}^{2}+\frac{\lambda}{2} \sigma_{\mu}^{2}\right]$ and expected utility of peripheral agents, $2(m-k)\left[u_{0}+\left(1-\frac{1}{m-k}\right) \lambda \sigma_{\varepsilon}^{2}\right]$, minus within and crossvillage link costs, $2 C+2 c[2(k-1)+2(m-k-1)]$. The welfare of a full-insurance-segregated network, $W_{F I S}$, is simply the expected utility from sharing idiosyncratic shocks within-village, $2 m\left[u_{0}+\left(1-\frac{1}{m}\right) \lambda \sigma_{\varepsilon}^{2}\right]$, minus link costs $2 c[2(m-1)]$ Comparing, we have $W_{C P} \geq W_{F I S}$ if and only if $k \lambda \sigma_{\mu}^{2}-\lambda \sigma_{\varepsilon}^{2} \geq 2 C-4 c$.

It then follows that if the village-shock variance is high enough, $\sigma_{\mu}^{2} \geq \frac{3}{2} \sigma_{\varepsilon}^{2}$, and the core is big enough, $k \geq 2$, for any values of the parameters where both graphs are stable, the core-periphery graph yields higher welfare.

But not all agents enjoy a gain. Agents in the periphery have lower expected utility in a core-periphery network than in a full-insurance network. They share idiosyncratic shocks with fewer people. But, core agents are better off in a core-periphery network, and their gains can outweigh the losses to the agents in the periphery.

\section{F. Different Size Villages}

Asymmetry in village sizes can strengthen the stability of networks with cross-village links. Consider $m_{1}>m_{2}$ and the following extension of core-periphery graphs. The larger village is divided

\footnotetext{
${ }^{15}$ When this is true, there are also regions where the core-periphery network is stable but the full-insurancesegregated networks are not for higher ranges of $c$.
} 
in two subgroups of sizes $m_{2}$ and $m_{1}-m_{2}$. The $m_{2}$ individuals in one subgroup are in the same component with the $m_{2}$ individuals of the smaller village, and with one link connecting both villages. The $m_{1}-m_{2}$ individuals in the other subgroup form a component; they are connected within but not across.

This network is pairwise stable when (a) the pair with the cross-village link does not want to

cut its link: $\left(\frac{1}{m_{2}}\right) \frac{\lambda}{2} \sigma_{\varepsilon}^{2}+\frac{\lambda}{2} \sigma_{\mu}^{2} \geq C$, and (b) in the larger village, core agents do not want to link with agents in the periphery: $\left(\frac{1}{2 m_{2}}-\frac{2}{m_{1}+m_{2}}\right) \frac{\lambda}{2} \sigma_{\varepsilon}^{2}-\left(\frac{m_{1}-m_{2}}{m_{1}+m_{2}}\right)^{2} \frac{\lambda}{2} \sigma_{\mu}^{2}<c$. The novel feature is that there is no counterpart to condition 1. Since the smaller village does not have a periphery, no lower bound has to be placed on $C$ to guarantee stability. Hence, this network can be stable for values of $C$ for which full insurance segregated networks are not stable.

\section{Conclusion}

This paper studies informal risk sharing across communities. We build a theoretical model where agents face idiosyncratic and community-level shocks. We find that networks that span communities can yield higher welfare than networks that connect all agents within a village. The gains to agents with a path to another community can outweigh the losses to those with no such path. Aggregate welfare increases, but some agents are worse off.

The paper provides a clear example where a network model gives us new insights. Individual incentives to form links drive the outcome. We could not see this outcome when decisions are made by the group or in some other cooperative way. We see how the individual costs and benefits from within versus cross-community links shape the pattern of risk sharing.

While we derive our results with very simple specifications of risk sharing and utility, the tradeoffs would hold in a general model. Pairwise stable networks would not necessarily involve minimally connected components. But there would still be an effect of cross-village links on the benefits of within village links. An individual would balance the gains from further insurance against idiosyncratic shocks with possible increase in variablility from sharing the village level shocks. We would also expect in general that the more individuals share the village shock, the less valuable is sharing of the idiosyncratic shock. The paper thus opens new questions on the nature of informal risk sharing, and we encourage further theoretical and empirical work to consider cross-community relations. 


\section{References}

Bloch, Francis, Genicot, Garance, and Ray, Debraj (2006): "Informal Insurance in Social Networks," working paper.

Bramoullé, Yann and Kranton, Rachel (2005): "Risk Sharing Networks," forthcoming Journal of Economic Behavior and Organization.

Coate, Stephen and Ravallion, Martin. (1993): "Reciprocity without Committment: Characterization and Performance of Informal Insurance Arrangements," Journal of Development Economics, 40, 1-24.

Dercon, Stefan and De Weerdt, Joachim. (2006): "Risk-Sharing Networks and Insurance against Illness", Journal of Development Economics 81(2), December 2006, pp. 337-356.

Fafchamps, Marcel and Lund, Susan (2003): "Risk Sharing Networks in Rural Philippines", Journal of Development Economics, 71, 261-87.

Jackson, M. (2003). "The Stability and Efficiency of Economic and Social Networks," in Networks and Groups: Models of Strategic Formation, edited by Bhaskar Dutta and Matthew O. Jackson, Springer-Verlag, Heidelberg 2003, p. 99-140.

Jackson, M., and A. Wolinsky (1996): "A Strategic Model of Social and Economic Networks," Journal of Economic Theory, 71, 44-74.

Ligon, E., Thomas, J., and Worrall, T. (2002): "Mutual Insurance and Limited Committment: Theory and Evidence in Village Economies," Review of Economic Studies, 69, 115-139.

Murgai, Rinku, Winters, Paul, Sadoulet, Elizabeth, and de Janvry, Alain. (2002): "Localized and incomplete mutual insurance," Journal of Development Economics, 67(2), 245274.

Rosenzweig, Mark R. and Stark, Oded. (1989) "Consumption Smoothing, Migration, and Marriage: Evidence from Rural India," The Journal of Political Economy, 97(4), August 1989, 905-926.

Townsend, Robert (1994): "Risk and Insurance in Village India", Econometrica, 62(3), May 1994, 539-591.

Udry, Christopher (1995): "Risk and Saving in Northern Nigeria," American Economic Review, 85(5), 1287-1300 